\title{
Ocular Manifestations of Systemic Sclerosis:A Descriptive Cross- Sectional Study from Iraq
}

\author{
${ }^{*}$ Zina Y. Jawad ${ }^{1}$, Faiq I. Gorial ${ }^{2}$ \\ ${ }^{1}$ Baghdad Teaching Hospital, Rheumatology Unit; Baghdad, Iraq \\ ${ }^{2}$ Department of Medicine, Collage of Medicine, University of Bagdad, Iraq \\ Corresponding Author: Faiq I. Gorial email:faiqig@gmail.com
}

\begin{abstract}
Background: Systemic sclerosis (scleroderma, SSc) is a rare severe systemic chronic connective tissue disease. Most of the data regardingocular involvement consist of single case reports or smallcase studies

Objectives: to evaluate the prevalence and characteristics of ocular manifestations in Iraqi patients with SSc.

Patients and methods: This cross-sectional study involved 50 patients with SSc diagnosed according to 2013 criteria developed by the American College of Rheumatology/European League Against Rheumatism for the classification of systemic sclerosis. Demographic and clinical data were recorded. Full ophthalmic examination was done for all patients.

Results: Prevalence of dry eye symptoms was 74\%, Keratoconjunctivitis sicca (KCS) was 68\%, eyelid skin stiffness and telangiectasia were observed in $56 \%$ and $36 \%$ respectively. Refractive errors were detected in about a quarter of cases (24\%). Myopia was the most frequently identified type of refractive errors (18\%), followed by astigmatism (6\%) and hypermetropia (only $2 \%$ after excluding the age dependent presbyopia). Blepharitis was diagnosed in $18 \%$ of examined patients. Subcapsular cataract, pterygium, conjunctival abnormalities, cataract-cortical type, uveitis (Anterior or posterior) and retinal microvascular abnormality were detected in less than $10 \%$ of cases.

Conclusions: Ocular manifestations in Iraqi patients with SSc were relatively common. The most common SScrelated ocular findings were dry eye symptoms, then KCS, followed by eyelid skin thickening and telangiectasia. Keywords: systemic sclerosis, scleroderma, ocular findings, ocular manifestations.
\end{abstract}

\section{Introduction}

Systemic sclerosis ( $\mathrm{SSc}$ ) is a severe chronic connective tissue disease caused by defect in immune system and altered structure and functions of blood vessels [1]. It is characterized by a pathological thickeningand tethering of the skin and involvement ofinternal organs [2]. Impairment of internal organs may lead to a wide spectrum of functional failures and limitations, affecting general health status and significantly decreasing health-related quality of life [3]. Ocular features in SSC may be related to the immune activation, vasculopathy, and excessive and widespread fibrosis [4].

Many ocular manifestations of SSc have been described, some being well-known associations and others reported for the first time in case reports [5]. Eyelid skin changes,Telangiectasia [6]. Conjunctival changes [6]. Shallowing of the fornices [7]. Keratoconjunctivitis sicca [7]. Eye dryness [8]. Pingueula and Pterygium [9] episcleritis, scleritis, uveitis, and peripheral ulcerative keratitis [10-12]. Other literature data showed an association between systemic sclerosis, and keratoconus and pellucid marginal degeneration [13]. Cataract [14]Glaucoma [15], Refractive error [16], Optic disk disease [17], and Retinal changes [18].Up to the best of our knowledge, there is no previous reports about prevalence of ocular manifestation in Iraqi patients with systemic sclerosis. This study aimed to evaluate the prevalence and characteristics of ocular manifestations in Iraqi patients with SSc.

\section{Study design}

\section{Patients and Methods}

This cross sectional study was conducted at the Rheumatology Unit, Department of Medicine in Baghdad Teaching Hospital from October 2014 to May 2015.Informed consent was obtained from each participant included in this study according to the declaration of Helsinki. Ethical approval was obtained from the Ethics Committee of College of Medicine, Medical Department, University of Baghdad. 


\section{Sample selection}

A total of 50 consecutive patients were included in the study diagnosed to have SSc according to 2013 American College of Rheumatology/European League Against Rheumatism for the classification of systemic sclerosis (19). Patients were excluded from the study if they had history of previous eye trauma, hypertension, diabetes mellitus, and overlap with other connective tissue diseases or inflammatory arthritis.

\section{Data collection and assessment}

Data recorded included patients baseline characteristics: ages, gender, duration of disease , disease subtype, and medications used. The study samples were checked for symptoms and signs of possible eye problem including: itching,burning, redness and dryness Data entry of patients were done using paper clinical research form (CRF) through interview and questionnaires. All the patients underwent complete ophthalmological examination by consultant ophthalmologist at Ophthalmology Unit in Al-Shaheed GhaziAlharery Teaching Hospital who is blinded to the diagnosis of SSc.

Ophthalmological examination involved visual acuity assessment with Snellen charts, examination of anterior and posterior eye segments with the slit lamp, Schirmer test and corneal fluorescein staining, and assessment of intraocular pressure with Goldmann applanation tonometer. Indirect ophthalmoscopy was performed with an indirect ophthalmoscope and a 20-diopter lens after both pupils were dilated with $1 \%$ tropicamide. The posterior pole was further evaluated by slit-lamp biomicroscopy using a 78-D Volk lens.

Blood investigations were measured in form of: Complete blood count (CBC), fasting blood sugar (FBS), renal function test (blood urea, serum creatinine), antinuclear antibody (ANA), anti-scleroderma 70 (AntiScl70), Anticentromere antibody, Anti-Sjögren's-syndrome-related antigen A (Anti-Ro) and AntiSjögren's-syndrome-related antigen B (Anti-La antibody).

\section{Statistical Analysis}

Statistical analyses were done using SPSS version 21. Frequency distributions for selected variables were done first. Categorical variables were presented as numbers and percentages.Compliance of quantitative random variables with normal distribution was analyzed using the Kolmogorov-Smirnov test. All the outcome (response) variables were normally distributed variables. Such variables were described by mean, standard deviation and standard error.

\section{Results}

A total of 50 patients with systemic sclerosis were involved in the study. The age of patients ranged

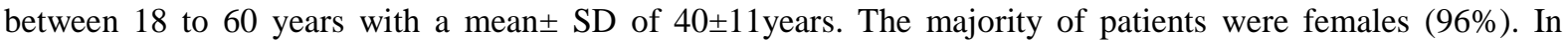
addition, the disease was categorized as limited in $56 \%$ and diffused in $44 \%$. Other patients' characteristics were shown in table1.As shown in table 2, the most frequently identified ocular manifestation in the study group was dry eye, observed in about three quarters (74\%) of subjects. Keratoconjunctivitis scicca ranked second in frequency among ocular problems, being identified in about two thirds (68\%) of subjects. Eyelid skin stiffness and eyelid telangiectasia were observed in $56 \%$ and $36 \%$ respectively. Refractive errors were detected in about a quarter of cases $(24 \%)$. Myopia was the most frequently identified type of refractive errors (18\%), followed by Astigmatism (6\%) and hypermetropia (only $2 \%$ after excluding the age dependent presbyopia). Blepharitis was diagnosed in $18 \%$ of examined patients. Each of the following ocular manifestations, namely: Subcapsular cataract, Pterygium, Conjunctivitis, Cataract-cortical type, Uveitis Present (Anterior or posterior) and Retinal microvascular abnormality were detected in less than $10 \%$ of cases.

Table 1: Baseline characteristics of 50 systemic sclerosis patients

\begin{tabular}{ll}
\hline Parameters & Values \\
\hline Age, Mean \pm SD, Range in years & $40 \pm 11,(18$ to 60$)$ \\
Females n (\%) & $48(96 \%)$ \\
Disease type n (\%) & $28(56 \%)$ \\
Limited & $22(44 \%)$ \\
Diffuse & \\
Drugs n(\%) & $32(64)$ \\
Steroids & $14(28)$ \\
Azathioprine & $12(24)$ \\
Methotrexate & $7(14)$ \\
Cyclophosphamide & $5(10)$ \\
Mycophenolate mofetil & $4(8)$ \\
Rituximab (Biologic) & $36(76.6)$ \\
Serum ANA Positive n(\%) & $17(38.6)$ \\
Serum anti-scl 70 antibodies Positive n(\%) & $6(14.3)$ \\
Serum Anti-centromere antibodies Positive n(\%) & \\
SD, standard deviation; ANA, antinuclear antibody; n, number; $\%$, percentage
\end{tabular}


Table 2: Relative frequency for selected ocular manifestations.

\begin{tabular}{llll}
\hline $\begin{array}{l}\text { Positive ocular manifestations } \\
\text { (Total n=50) }\end{array}$ & n & \% & $\mathbf{9 5 \%}$ CI \\
\hline Dry eyes & 37 & 74.0 & $(59.7 \%$ to $85.7 \%)$ \\
Kerato-conjunctivitis scicca & 34 & 68.0 & $(53.3 \%$ to $80.5 \%)$ \\
Eyelid skin stiffness & 28 & 56.0 & $(41.3 \%$ to $70.0 \%)$ \\
Eyelid telangiectasia & 18 & 36.0 & $(22.9 \%$ to $50.8 \%)$ \\
Refractive errors (any type) & 12 & 24.0 & $(13.1 \%$ to $38.2 \%)$ \\
$\quad$ Myopia & 9 & 18.0 & $(8.6 \%$ to $31.4 \%)$ \\
$\quad 3 s t i g m a t i s m$ & 3 & 6.0 & $(1.3 \%$ to $16.6 \%)$ \\
$\quad$ Hypermetropia (excluding presbyopia) & 1 & 2.0 & $(0.1 \%$ to $10.7 \%)$ \\
Blepheritis & 9 & 18.0 & $(8.6 \%$ to $31.4 \%)$ \\
Subcapsular cataract & 5 & 10.0 & $(3.3 \%$ to $21.8 \%)$ \\
Pterygium & 4 & 8.0 & $(2.2 \%$ to $19.2 \%)$ \\
Conjunctivitis & 4 & 8.0 & $(2.2 \%$ to $19.2 \%)$ \\
Cataract-cortical type & 4 & 8.0 & $(2.2 \%$ to $19.2 \%)$ \\
Uveitis Present ( Ant or post) & 2 & 4.0 & $(0.5 \%$ to $13.7 \%)$ \\
Retinal microvascular abnormality & 1 & 2.0 & $(0.1 \%$ to $10.7 \%)$ \\
\hline
\end{tabular}

n, number, \%, percentage; CI, confidence interval; Ant., anterior; post, posterior

\section{Discussion}

There are few reports available concerning ophthalmological complications in the course of systemic sclerosis. Up to the best of our knowledge, this the first report that assessed the prevalence and characteristics of ocular manifestation in Iraqi patients with SSc.The most frequently identified ocular manifestation in this study group was dry eye symptoms (74\%). Dry eye syndrome is believed to be caused by fibrosis-related impairment of lacrimal gland secretion, namely water portion of the tear film or lipid layer disorder due to chronic blepharitis and Meibomian gland dysfunction, as well as increased evaporation of tears from the ocular surface in the case of restricted eyelid mobility and thus reduced blinking [20]. This finding is comparable with previous studies that showed 34.6-72\% of patients with SSc have ocular symptoms [21,22].

Keratoconjunctivitis sicca (KCS) was present in $3468 \%$ ) patients which is within the range of previous study that showeda prevalence ranging from $37-79 \%$ of patients[5].The current study reported that eyelid skin changes were seen in $56 \%$ of the patients. The fibrotic changes seen in the eyelids may be explained by excessive deposition and accumulation of extracellular matrix, mainly type I collagen, in the dermis [23]. Similarly, other studies have reported variable prevalence of eyelid changes ranged from 29 to $65 \%$ of patients $[5,24]$. In this study, Telangiectasia was observed in $36 \%$ of patients which is lower than that found in Gomes et al study (51.1\%) [25]. This may be due to different in sample size. The finding of refractive error was $24 \%$ where myopia was (18\%), followed by astigmatism (6\%) and hypermetropia (only $2 \%$ ). Refractory errors may be explained by corneal thickening. Significant increase of corneal thickness occurs in early stages of SSc. In Gomes et al. (25) study, astigmatism was present in $2.2 \%$ of patients. Another study done by Waszczykowska A et al [26] demonstrated that astigmatism occurred in over half of the eyes and was significantly more commonly than in the control group. These variations may be explained by the different genetic factors and difference in sample size between the two populations. The prevalence of blepharitis found in this study was $18 \%$ and that of pterygium 8\% which were less than the result of Gomes et al (blepharitis $40 \%$ and pterygium $15.6 \%$ [25]. This may be related to the different genetic and environmental factors.

In the present study, cataracts at various stages were observed in about $18 \%$ of the patients, posterior subcapsular was the most common form 10\% then cortical cataract $8 \%$. Incidence of this cataract form in SSc patients is believed to result from lens nutrition disorders secondary to narrowing of ciliary body vessels and/or complications of general steroid treatment [13, 14].

These findings were lower than the result of Waszczykowska et al [26] who reported that cataract were present in $50 \%$ of their patients. This disagreement may be explained by the larger number of patients enrolled in this study as well as the genetic factors.

Conjunctival abnormality was observed in $8 \%$ of our patients (4\% had segmented tortuous conjunctival vessels and $4 \%$ had allergic conjunctivitis) whereas another study [25] found conjunctival vascular congestion (8.9\%) and a loss of fine conjunctival vessels (4.4\%). This disagreement may be due to sample size and differences in genetic and environmental factors between the two populations.

In the current study, tow patients had a history of past inactive uveitis. Indeed, the association between SSc and uveitis is rare, and only single case has been reported in the peer-reviewed literature [27]. Also Waszczykowska et al [26] found one patient had a history of past recurrent uveitis. Retinal microvascular abnormality was noted in only one patient (2\%) whereas it was found in 13 patients (28.9\%) in another study [25]. This disagreement may be due to inter-observers' variations and difference in demographic features of the study population.

Ushiyama et al [28] evaluated the retinal findings of 29 patients with SSc and showed a higher frequency of retinal changes associated with vascular damage in SSc patients than in controls. Possibly, 
thevasculopathy that occurs in SSc may play a role in the development of retinal vascular abnormalities and may contribute to retinal vascular occlusions

The main limitation of this study is the small sample size due to short period of the study and rare disease. This may be solved by a larger and longer duration prospective study. However, this study has strict inclusion and exclusion criteria, and data collection and measurements in addition to be the first study up to the best of our knowledge in Iraq that assessed the ocular manifestation of SSc.

In conclusion, ocular manifestations of SSc were relatively common, Dry eye symptoms and KCS were the most common SSc-related ocular findings. Rank Next was eyelid skin thickening and telangiectasia. Then the Refractive errors, and subsequently the other ocular findings. This suggests early and frequent eye monitoring for patients with SSc will help in early diagnosis and treatment and consequently prevention of serious complications.

\section{References}

[1] Dziankowska-Bartkowiak B, Gerlicz-Kowalczuk Z, Waszczy -kowska E. Angiogenin and SDF-1alpha serum concen -tration in patients with systemic sclerosis in relation to clinical status. Arch Med Sci 2011; 1: 92-6.

[2] Chifflot H, Fautrel B, Sordet C, et al. Incidence and prevalence of systemic sclerosis: a systematic literature review. Semin Arthritis Rheum 2008; 37:223-35.

[3] Hudson M, Thombs BD, Steele R , et al. Canadian Scleroderma Research Group Health-related quality of life in systemic sclerosis: a systematic review. Arthritis Rheum 2009; 61:1112-20.

[4] Sakkas LI. New developments in the pathogenesis of systemic sclerosis. Autoimmunity. 2005;38:113-6.

[5] Tailor R, Gupta A, Herrick A, Kwartz J. Ocular manifestations of scleroderma. Surv Ophthalmol 2009; 54:292-304, doi: 10.1016/ j. survophthal. 2008.12.007.)

[6] West RH, Barnett AJ. Ocular involvement in scleroderma. Br J Ophthalmol. 1979;63: 845-7.

[7] Kirkham TH. Scleroderma and Sjogren's syndrome. Br J Ophthalmol. 1969;53:131-3.

[8] Wases B AF, Santhiago MR, Magalha es P, et al. Ocular findings in patients with systemic sclerosis. CLINICS 2011;66(3):379-85.

[9] Kanski JJ, Bowling B. Clinical Ophthalmology: A Systematic Approach. 7th ed. Philadelphia: Elsevier/Saunders; 2011:219-20.

[10] Hamideh F, Prete PE. Ophthalmologic manifestations of rheumatic diseases.Semin Arthritis Rheum. 2001; 30:217-41, doi: 10.1053/sarh.2001. 16639.

[11] De Andre's J, Garci'a-Delpech S, Pe'rez VL, et al. Bilateral infusion pump implants as therapy for refractory corneal ulcers in a patient with CREST syndrome: an interdisciplinary approach. Arch Ophthalmol. 2008; 126:964-7, doi: 10.1001/ archopht.126.7.964.

[12] Sii F, Lee GA, Sanfilippo P, Stephensen DC. Pellucid marginal degeneration and scleroderma. Clin Exp Optom. 2004;87:180-4.

[13] Pastuszka M, Kaszuba A. Status of combination drugs with betamethasone dipropionate and salicylic acid in the treatment of skin diseases. Postep Derm Alergol. 2012; 29:196-204.

[14] Dańczak-Pazdrowska A. Place of methotrexate in the treatment of psoriasis in the era of biologic agents. Postep Derm Alergol. 2012;29:182-8.

[15] Sakata K, Sakata LM, Sakata VM, et al. Prevalence of glaucoma in a South brazilian population: Projeto Glaucoma. Invest Ophthalmol Vis Sci. 2007; 48:4974-9, doi: 10.1167/iovs. 07-0342.

[16] Allanore Y, Parc C, Monnet D, et al. Increased prevalence of ocular glaucomatous abnormalities in systemic sclerosis. Ann Rheum Dis 2004; 63: 1276-8.

[17] Boschi A, Snyers B, Lambert M. Bilateral optic neuropathy associated with the crest variant of scleroderma. Eur J Ophthalmol. $1993 ; 3: 219-22$.

[18] Ashton N, Coomes EN, Garner A, Oliver DO. Retinopathy due to progressive systemic sclerosis. J Path. 1968; 96:259-68.

[19] Hoogen FV, Khanna D, Fransen J, et al. 2013 Classification Criteria for Systemic Sclerosis. Arthr. \& Rheu.2013; 1-11. DOI 10.1002/art.38098.

[20] Waszczykowska A , Goś R , Waszczykowska E et al. Prevalence of ocular manifestations in systemic sclerosis patients, Arch Med Sci 2013; 9, 6: 1107-1113.

[21] Rasker JJ, Jayson MI, Jones DE, et al . Sjögren's syndrome in systemic sclerosis. A clinical study of 26 patients. Scand J Rheumatol $1990 ; 19: 57-65$.

[22] Avouac J, Sordet C, Depinay C, et al Systemic sclerosis-associated Sjögren's syndrome and relationship to the limited cutaneous subtype: results of a prospective study of sicca syndrome in 133 consecutive patients. Arthritis Rheum 2006; 54:2243-2249.

[23] Jinnin M. Mechanisms of skin fibrosis in systemic sclerosis. J Dermatol 2010; 37:11-25.

[24] Choudhary MM, Hajj-Ali RA, Lowder CY. Gender and Ocular Manifestations of Connective Tissue Diseases and Systemic Vasculitides. J Ophthalmol. 2014;2014:1-8.

[25] Waszczykowska A, Goś R, Waszczykowska E et al. Prevalence of ocular manifestations in systemic sclerosis patients, Arch Med Sci 2013; 9, 6: 1107-1113.

[26] Akman A, Akova YA, Yu“ cel E, Aydin P. Granulomatous anterior uveitis in a patient with CREST syndrome.Ocul Immunol Inflamm. 2000;8:201-3.

[27] Ushiyama O, Ushiyama K , Yamada T et al . Retinal findings in systemic sclerosis: a comparison with nailfold capillaroscopic patterns. Ann Rheum Dis 2003;62:204-207.

[28] Gomes B AF, Santhiago MR, Magalha es P, et al. Ocular findings in patients with systemic sclerosis. CLINICS 2011;66(3):37985.

Zina Y. Jawad . "Ocular Manifestations of Systemic Sclerosis: A Descriptive Cross-Sectional Study from Iraq." IOSR Journal of Nursing and Health Science (IOSR-JNHS) 6.4 (2017): 94-97. 